University of Nebraska - Lincoln

DigitalCommons@University of Nebraska - Lincoln

John R. Hardy Papers

Research Papers in Physics and Astronomy

7-15-1975

\title{
Theoretical Study of Impurity-Induced First-Order Raman Spectra for the Alkali Halides
}

Arnold Karo

University of Nebraska - Lincoln

John R. Hardy

University of Nebraska - Lincoln

Follow this and additional works at: https://digitalcommons.unl.edu/physicshardy

Part of the Physics Commons

Karo, Arnold and Hardy, John R., "Theoretical Study of Impurity-Induced First-Order Raman Spectra for the Alkali Halides" (1975). John R. Hardy Papers. 29.

https://digitalcommons.unl.edu/physicshardy/29

This Article is brought to you for free and open access by the Research Papers in Physics and Astronomy at DigitalCommons@University of Nebraska - Lincoln. It has been accepted for inclusion in John R. Hardy Papers by an authorized administrator of DigitalCommons@University of Nebraska - Lincoln. 


\title{
Theoretical study of impurity-induced first-order Raman spectra for the alkali halides*
}

\author{
Arnold M. Karo and John R. Hardy ${ }^{\dagger}$ \\ Lawrence Livermore Laboratory, University of California, Livermore, California 94550 \\ (Received 27 January 1975)
}

\begin{abstract}
A deformation-dipole model with first- and second-neighbor central short-range forces is used as a basis for calculating all symmetry-adapted combinations of eigenvectors (SACEV) necessary for the study of defect vibrations in alkali-halide crystals. As an example of the full set of spectral weight functions, which are tabulated separately, sample results are given for the predicted defect-activated $E_{\mathrm{g}}$ Raman spectra for $\mathrm{KCl}, \mathrm{KI}$, $\mathrm{RbCl}$, and $\mathrm{KBr}$, assuming no force-constant changes and that the defect is located at a postive-ion site. The results show qualitiative agreement with the earlier findings of Harley, Page, and Walker, even though the deformation-dipole model is not adjusted to fit measured dispersion curves. The SACEV compilations, therefore, should be helpful to other researchers initiating defect studies and needing first-trial solutions to use as a basis for subsequent, more refined theoretical investigations.
\end{abstract}

\section{INTRODUCTION}

Theoretical studies on the lattice dynamics of specific materials often culminate in predictions of phonon dispersion curves and frequency spectra. The former type of prediction generally can be tested by comparison with experimental data obtained by inelastic neutron scattering; however, there is no direct experimental check on the latter. This is unfortunate, since frequency spectra, by definition, contain information on all phonons, while dispersion curves provide information on only a very restricted set. Experimental studies of defect-activated infrared absorption provide indirect information on the dynamics of the host lattice, but the reliability of this information depends critically on an accurate knowledge of how a given defect perturbs the lattice; this information is seldom available. Indeed, the general philosophy of such studies has been to use a lattice-dynamical model that gives a good fit to the measured phonon dispersion curves, assume that it is equally good for all phonons, and then, on this basis, construct the best possible model of the defect.

In 1971 Harley, Page, and Walker ${ }^{1}$ (hereafter referred to as HPW) presented the results of studies on defect-activated single-phonon Raman scattering from $\mathrm{Tl}^{+}$-doped $\mathrm{KCl}, \mathrm{KBr}, \mathrm{KI}$, and $\mathrm{RbCl}$. Their results indicated that here, for the first time, was a means of obtaining a direct map of the projections of the perfect-lattice modes on the Raman-active $A_{1 g}, E_{g}$, and $T_{2 g}$ vibrations of the octahedron formed by the six first neighbors of the $\mathrm{Tl}^{+}$ion. Moreover, since perfect crystals show no firstorder Raman effect, these maps can cover the whole range spanned by their frequency spectra. Infrared measurements cannot be extended into the region of the optical branches of the spectra because defect-activated absorption in this frequency range is overshadowed by the intrinsic absorption of the host lattice.
The HPW experiments used breathing-shell models fitted to known phonon dispersion curves. In this study, deformation-dipole models ${ }^{2,3}$ are used which include central, short-range forces acting between first-neighbor ions and second-neighbor negative ions. No attempt has been made to fit the measured dispersion curves.

The primary objective of the investigation is to demonstrate a method for using spectroscopic data to test a given model and identify its shortcomings without reference to other measurements. The secondary purpose is to call attention to the fact that, in collaboration with a University of Reading

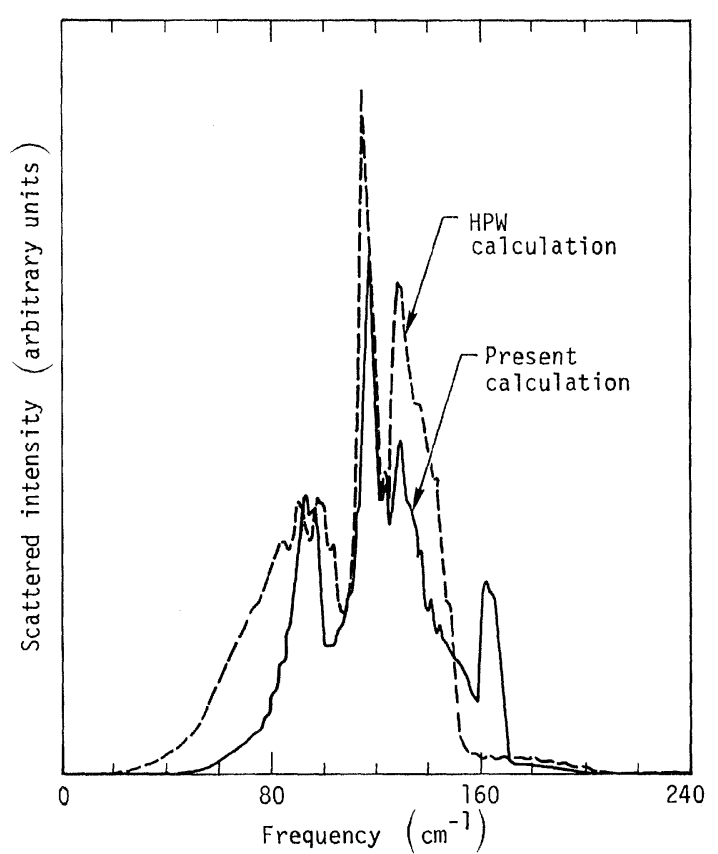

FIG. 1. Theoretical $E_{\boldsymbol{g}}$ compoennts of the defect-activated Raman spectrum for $\mathrm{KCl}$, assuming no force-constant changes for a substitutional monovalent impurity (e.g., $\mathrm{Tl}^{+}$) at the $\mathrm{K}^{+}$-ion site. 


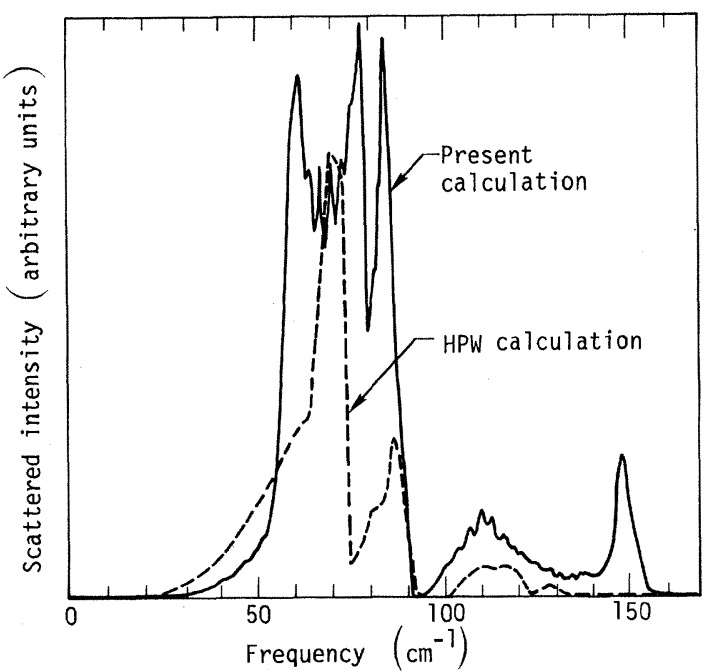

FIG. 2. Theoretical $E_{\text {g }}$ components of the defect-activated Raman spectrum for $\mathrm{KBr}$, assuming no force-constant changes for a substitutional monovalent impurity (e. g. , $\mathrm{Tl}^{+}$) at the $\mathrm{K}^{+}$-ion site.

research group headed by Professor C. W. McCombie, we have compiled a systematic listing of all possible densities of states for every $\mathrm{NaCl}-$ structured alkali halide. These data, tabulated for ease of key punching onto data cards, can be used in defect calculations for any of these crystals. This compilation offers two advantages: (i) The user does not have to write and debug his own latticedynamics program; (ii) the data, generated using a mesh of 64000 sample wave vectors in the first

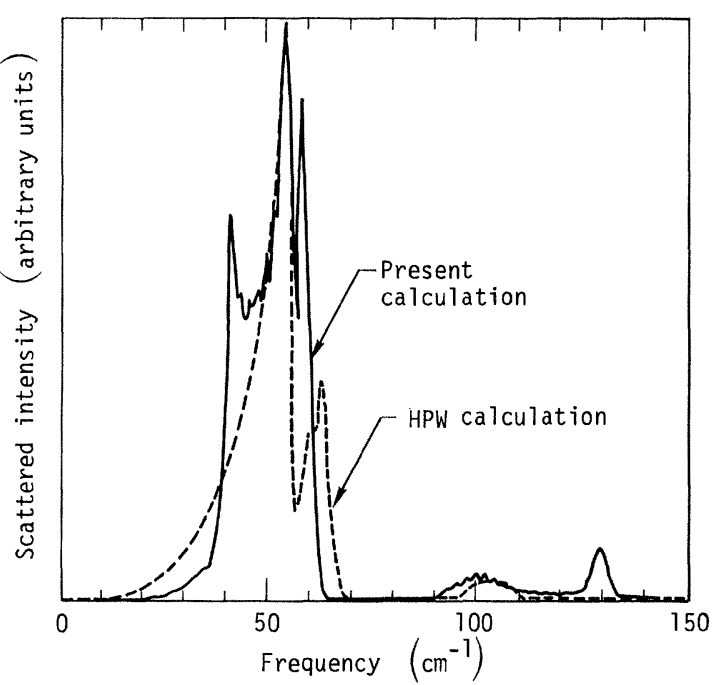

FIG.3. Theoretical $E_{\boldsymbol{g}}$ components of the defect-activated Raman spectrum for KI, assuming no force-constant changes for a substitutional monovalent impurity (e.g., $\mathrm{Tl}^{+}$) at the $\mathrm{K}^{+}$-ion site.

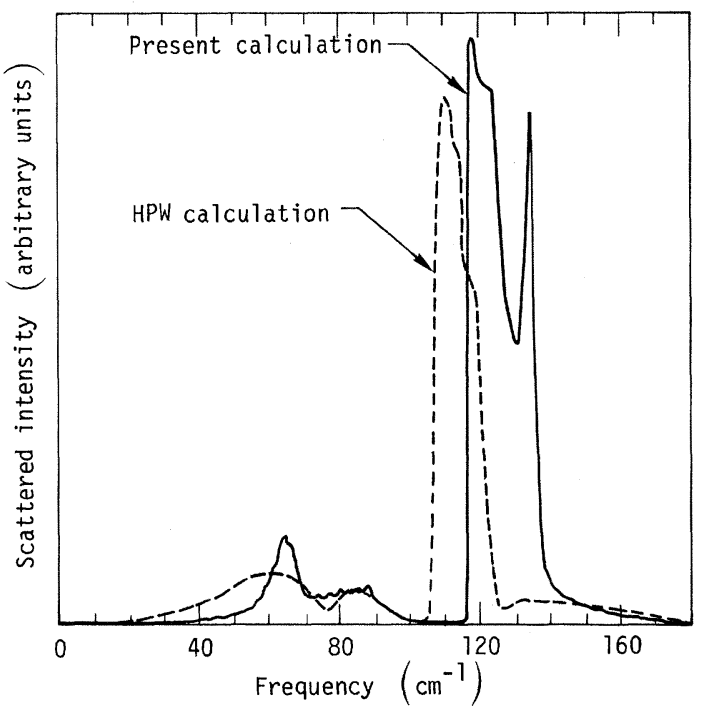

FIG. 4. Theoretical $E_{g}$ components of the defect-activated Raman spectrum for $\mathrm{RbCl}$, assuming no force-constant changes for a substitutional monovalent impurity (e.g., $\mathrm{Tl}^{+}$) at the $\mathrm{Rb}^{+}$-ion site.

Brillouin zone, can be used directly by researchers with limited computational facilities. ${ }^{4}$

\section{GENERAL THEORY}

McCombie's approach ${ }^{5}$ to the study of the dynamics of imperfect crystals is less formal and more physical than the more standard method of using Green's functions although the final results of both approaches are identical. In the McCombie approach, the basic problem is to determine the response of the imperfect lattice to an external "force" $F=\underline{F}_{0} e^{i \omega t}$, which is assumed to couple only to the motion of the impurity and its six first neighbors. The 21 independent components of the displacements of these seven ions can be analyzed into the normal modes of this octahedral complex which we denote by $x_{s}$. Each of these, in turn, can be analyzed into the normal modes of the perfect lattice, designated $\underline{Q}(\vec{q}, j)$, where $2 \pi \vec{q}$ is the phonon wave vector and $\vec{j}$ is the branch index. Thus,

$$
\underline{x}_{s}=\sum_{\overrightarrow{\mathrm{q}}, j} \alpha_{s}(\overrightarrow{\mathrm{q}}, j) \underline{Q}(\overrightarrow{\mathrm{q}}, j) .
$$

Similarly, we can analyze the force $F$ into generalized components:

$$
\underline{F}=\sum_{\vec{q}, j, t} \alpha_{t}(\overrightarrow{\mathrm{q}}, j) \underline{F}_{t} .
$$

Then, allowing for dissipation, the forced amplitude of the mode $\vec{q}, j$ is given by

$$
\underline{Q}(\overrightarrow{\mathrm{q}}, j)=\sum_{r} \frac{\alpha_{t}(\overrightarrow{\mathrm{q}}, j)}{\omega^{2}(\overrightarrow{\mathrm{q}}, j)-\omega^{2}+i \epsilon \omega} F_{t} .
$$

Hence, 


$$
x_{s}=\sum_{\overrightarrow{\mathrm{q}}, j, t} \frac{\alpha_{s}(\overrightarrow{\mathrm{q}}, j) \alpha_{t}(\overrightarrow{\mathrm{q}}, j)}{\omega^{2}(\overrightarrow{\mathrm{q}}, j)-\omega^{2}+i \epsilon \omega} \underline{F}_{t} .
$$

These equations apply to a perfect lattice. If a lattice contains an impurity at the center of the octahedron, the dynamical response of the lattice is changed. However, if the associated perturbation is assumed to be restricted to the central atom plus its first-neighbor bonds, it can be expressed formally as an effective change in driving force as follows:

$$
\delta \underline{F}_{t}=\sum_{r} \Delta_{t r} \underline{x}_{r}
$$

where the matrix elements $\Delta_{t r}$ depend on the nature of the defect. The matrix $\Delta$ and Eq. (5) specify the effect of the imperfection completely, since $\Delta$ has no elements which couple the subspace of vectors $\underline{x}$ to any other coordinates. Thus, combining Eqs.

(4) and (5) we obtain

$$
\underline{x}_{s}=\sum_{\overrightarrow{\mathrm{q}}, j, r, t} \frac{\alpha_{s}(\overrightarrow{\mathrm{q}}, j) \alpha_{t}(\overrightarrow{\mathrm{q}}, j)}{\omega^{2}(\overrightarrow{\mathrm{q}}, j)-\omega^{2}+i \epsilon \omega}\left(\underline{F}_{t}+\Delta_{t r} \underline{x}_{r}\right) .
$$

Evidently, these inhomogeneous simultaneous equations can be used to express each $x_{s}$ in terms of the true external driving forces $F_{t}$. This means the average power absorbed or emitted, as measured by

$$
\operatorname{Im}\left(\sum_{s} \underline{F}_{s} \cdot \underline{x}_{s}\right)_{\mathrm{av}}
$$

[where the ()$_{\text {av }}$ denotes time average], can be computed as a function of the driving frequency $\omega$.

Thus far in our analysis we have made no explicit use of symmetry arguments. The coefficients $\alpha$ could project the normal modes of the crystal onto arbitrary sets of combinations of the nearestneighbor displacements. However, if the normal coordinates of the octahedral complex are classified according to the irreducible representations of the $O_{h}$ point group, the following possibilities result:

$$
A_{1 g}, E_{g}, T_{1 g}, T_{2 g}, T_{1 u} \text {, and } T_{2 u} \text {. }
$$

When this classification is used, only the following products of $\alpha_{s}(\overrightarrow{\mathrm{q}}, j) \alpha_{t}(\overrightarrow{\mathrm{q}}, j)$ can appear in Eq. (6):

$$
\begin{aligned}
& A_{1 g}(r=s=1): \\
& {\left[\alpha_{1}(\overrightarrow{\mathrm{q}}, j)\right]^{2}=4\left(\sum_{\alpha} W(k \mid \overrightarrow{\mathrm{q}}, j)_{\alpha} \sin \left(\pi \overrightarrow{\mathrm{q}}_{\alpha} r_{0}\right)\right)^{2}} \\
& +\left[W(k \mid \overrightarrow{\mathrm{q}}, j)_{y} \sin \left(\pi \overrightarrow{\mathrm{q}}_{y} r_{0}\right)-W(k \mid \overrightarrow{\mathrm{q}}, j)_{z} \sin \left(\pi \overrightarrow{\mathrm{q}}_{z} r_{0}\right)\right]^{2} \\
& \left.+\left[W(k \mid \overrightarrow{\mathrm{q}}, j)_{z} \sin \left(\pi \overrightarrow{\mathrm{q}}_{z} r_{0}\right)-W(k \mid \overrightarrow{\mathrm{q}}, j)_{x} \sin \left(\pi \overrightarrow{\mathrm{q}}_{x} r_{0}\right)\right]^{2}\right\} ; \\
& {\left[\alpha_{3}(\overrightarrow{\mathrm{q}}, j)\right]^{2}=2\left\{\left[W(k \mid \overrightarrow{\mathrm{q}}, j)_{x} \sin \left(\pi \overrightarrow{\mathrm{q}}_{y} r_{0}\right)-W(k \mid \overrightarrow{\mathrm{q}}, j)_{y} \sin \left(\pi \overrightarrow{\mathrm{q}}_{x} r_{0}\right)\right]^{2}\right.} \\
& +\left[W(k \mid \overrightarrow{\mathrm{q}}, j)_{y} \sin \left(\pi \overrightarrow{\mathrm{q}}_{z} r_{0}\right)-W(k \mid \overrightarrow{\mathrm{q}}, j)_{z} \sin \left(\pi \overrightarrow{\mathrm{q}}_{y} r_{0}\right)\right]^{2} \\
& \left.+\left[W(k \mid \overrightarrow{\mathrm{q}}, j)_{z} \sin \left(\pi \overrightarrow{\mathrm{q}}_{x} r_{0}\right)-W(k \mid \overrightarrow{\mathrm{q}}, j)_{x} \sin \left(\pi \overrightarrow{\mathrm{q}}_{z} r_{0}\right)\right]^{2}\right\},
\end{aligned}
$$

$T_{2 g}(r=s=4)$ :

$$
\begin{aligned}
{\left[\alpha_{4}(\overrightarrow{\mathrm{q}}, j)\right]^{2}=} & 2\left\{\left[W(k \mid \overrightarrow{\mathrm{q}}, j)_{x} \sin \left(\pi \overrightarrow{\mathrm{q}}_{y} r_{0}\right)+W(k \mid \overrightarrow{\mathrm{q}}, j)_{y} \sin \left(\pi \overrightarrow{\mathrm{q}}_{x} r_{0}\right)\right]^{2}\right. \\
& +\left[W(k \mid \overrightarrow{\mathrm{q}}, j)_{y} \sin \left(\pi \overrightarrow{\mathrm{q}}_{z} r_{0}\right)+W(k \mid \overrightarrow{\mathrm{q}}, j)_{z} \sin \left(\pi \overrightarrow{\mathrm{q}}_{y} r_{0}\right)\right]^{2} \\
& \left.+\left[W(k \mid \overrightarrow{\mathrm{q}}, j)_{z} \sin \left(\pi \overrightarrow{\mathrm{q}}_{x} r_{0}\right)+W(k \mid \overrightarrow{\mathrm{q}}, j)_{x} \sin \left(\pi \overrightarrow{\mathrm{q}}_{z} r_{0}\right)\right]^{2}\right\} ;
\end{aligned}
$$

$T_{1 u}(1) \quad(r=s=5):$

$$
\left[\alpha_{5}(\overrightarrow{\mathrm{q}}, j)\right]^{2}=4\left(\sum_{\alpha} W(k \mid \overrightarrow{\mathrm{q}}, j)_{\alpha} \cos \left(\pi \overrightarrow{\mathrm{q}}_{\alpha} r_{0}\right)\right)^{2} ;
$$

$$
\begin{aligned}
T_{1 u}(2) \quad(r=s= & 6): \\
{\left[\alpha_{6}(\overrightarrow{\mathrm{q}}, j)\right]^{2}=} & 2\left(\left\{W(k \mid \overrightarrow{\mathrm{q}}, j)_{x}\left[\cos \left(\pi \overrightarrow{\mathrm{q}}_{y} r_{0}\right)+\cos \left(\pi \overrightarrow{\mathrm{q}}_{z} r_{0}\right)\right]\right\}^{2}\right. \\
& +\left\{W(k \mid \overrightarrow{\mathrm{q}}, j)_{y}\left[\cos \left(\pi \overrightarrow{\mathrm{q}}_{z} r_{0}\right)+\cos \left(\pi \overrightarrow{\mathrm{q}}_{x} r_{0}\right)\right]\right\}^{2} \\
& \left.+\left\{W(k \mid \overrightarrow{\mathrm{q}}, j)_{z}\left[\cos \left(\pi \overrightarrow{\mathrm{q}}_{x} r_{0}\right)+\cos \left(\pi \overrightarrow{\mathrm{q}}_{y} r_{0}\right)\right]\right\}^{2}\right),
\end{aligned}
$$




$$
\begin{aligned}
& T_{2 u}(r=s=7): \\
& {\left[\alpha_{7}(\overrightarrow{\mathrm{q}}, j)\right]^{2}=} 2\left(\left\{W(k \mid \overrightarrow{\mathrm{q}}, j)_{x}\left[\cos \left(\pi \overrightarrow{\mathrm{q}}_{y} r_{0}\right)-\cos \left(\pi \overrightarrow{\mathrm{q}}_{z} r_{0}\right)\right]\right\}^{2}\right. \\
&+\left\{W(k \mid \overrightarrow{\mathrm{q}}, j)_{y}\left[\cos \left(\pi \overrightarrow{\mathrm{q}}_{z} r_{0}\right)-\cos \left(\pi \overrightarrow{\mathrm{q}}_{x} r_{0}\right)\right]\right\}^{2} \\
&\left.+\left\{W(k \mid \overrightarrow{\mathrm{q}}, j)_{z}\left[\cos \left(\pi \overrightarrow{\mathrm{q}}_{x} r_{0}\right)-\cos \left(\pi \overrightarrow{\mathrm{q}}_{y} r_{0}\right)\right]\right\}^{2}\right) ;
\end{aligned}
$$

$T_{1 u}(r=s=8)$ :

$$
\left[\alpha_{8}\left(\overrightarrow{\mathrm{q}}_{j}\right)\right]^{2}=2\left(\sum_{\alpha} W(k \mid \overrightarrow{\mathrm{q}}, j)_{\alpha}\right)^{2}
$$

and finally, three cross terms that mix different $\alpha$ 's having the same symmetry,

$$
\begin{aligned}
T_{1 u}(r=5 ; s=6): & \\
\alpha_{5}(\overrightarrow{\mathrm{q}}, j) \alpha_{6}(\overrightarrow{\mathrm{q}}, j)= & 2 \sqrt{2}\left(\left[W(k \mid \overrightarrow{\mathrm{q}}, j)_{x}\right]^{2}\left\{\cos \left(\pi \overrightarrow{\mathrm{q}}_{x} r_{0}\right)\left[\cos \left(\pi \overrightarrow{\mathrm{q}}_{y} r_{0}\right)+\cos \left(\pi \overrightarrow{\mathrm{q}}_{z} r_{0}\right)\right]\right\}\right. \\
& +\left[W(k \mid \overrightarrow{\mathrm{q}}, j)_{y}\right]^{2}\left\{\cos \left(\pi \overrightarrow{\mathrm{q}}_{y} r_{0}\right)\left[\cos \left(\pi \overrightarrow{\mathrm{q}}_{z} r_{0}\right)+\cos \left(\pi \overrightarrow{\mathrm{q}}_{x} r_{0}\right)\right]\right\} \\
& \left.+\left[W(k \mid \overrightarrow{\mathrm{q}}, j)_{z}\right]^{2}\left\{\cos \left(\pi \overrightarrow{\mathrm{q}}_{z} r_{0}\right)\left[\cos \left(\pi \overrightarrow{\mathrm{q}}_{x} r_{0}\right)+\cos \left(\pi \overrightarrow{\mathrm{q}}_{y} r_{0}\right)\right]\right\}\right) ;
\end{aligned}
$$

$T_{1 u}(r=5 ; s=8)$ :

$$
\alpha_{5}(\overrightarrow{\mathrm{q}}, j) \alpha_{8}(\overrightarrow{\mathrm{q}}, j)=2 \sqrt{2}\left[\sum_{\alpha} W(k \mid \overrightarrow{\mathrm{q}}, j)_{\alpha} W\left(k^{\prime} \mid \overrightarrow{\mathrm{q}}, j\right)_{\alpha} \cos \left(\pi \overrightarrow{\mathrm{q}}_{\alpha} r_{0}\right)\right] \quad\left(k \neq k^{\prime}\right) ;
$$

and

$$
\begin{aligned}
T_{1 u}(r=6 ; s=8): & \\
\alpha_{6}(\overrightarrow{\mathrm{q}}, j) \alpha_{8}(\overrightarrow{\mathrm{q}}, j)= & 2\left\{\left[W(k \mid \overrightarrow{\mathrm{q}}, j)_{x} W\left(k^{\prime} \mid \overrightarrow{\mathrm{q}}, j\right)_{x}\right]\left[\cos \left(\pi \overrightarrow{\mathrm{q}}_{y} r_{0}\right)+\cos \left(\pi \overrightarrow{\mathrm{q}}_{z} r_{0}\right)\right]\right. \\
& +\left[W(k \mid \overrightarrow{\mathrm{q}}, j)_{y} W\left(k^{\prime} \mid \overrightarrow{\mathrm{q}}, j\right)_{y}\right]\left[\cos \left(\pi \overrightarrow{\mathrm{q}}_{z} r_{0}\right)+\cos \left(\pi \overrightarrow{\mathrm{q}}_{x} r_{0}\right)\right] \\
& +\left[W(k \mid \overrightarrow{\mathrm{q}}, j)_{z} W\left(k^{\prime} \mid \overrightarrow{\mathrm{q}}, j\right)_{z}\right]\left[\cos \left(\pi \overrightarrow{\mathrm{q}}_{x} r_{0}\right)+\cos \left(\pi \overrightarrow{\mathrm{q}}_{y} r_{0}\right)\right] \quad\left(k \neq k^{\prime}\right) .
\end{aligned}
$$

In Eqs. (7)-(17), the $W$ 's are eigenvector components, each divided by the square root of the appropriate mass; $r_{0}$ is the nearest-neighbor distance and $k$ and $k^{\prime}$ index the two sublattices.

For comparison with experiment one needs the corresponding spectral functions appropriate to an infinite crystal. These are defined as follows:

$$
\begin{aligned}
A_{r s}(\omega)= & \lim _{\Delta \omega \rightarrow 0, N \rightarrow \infty} \frac{1}{6 N \Delta \omega} \sum_{\vec{q} j} \int_{\omega}^{\omega+\Delta \omega} \alpha_{r}(\overrightarrow{\mathrm{q}}, j) \alpha_{s}(\overrightarrow{\mathrm{q}}, j) \\
& \times \delta\left(\omega^{\prime}-\omega(\overrightarrow{\mathrm{q}}, j)\right) d \omega^{\prime},
\end{aligned}
$$

where $N$ is the number of unit cells in the crystal. The response for a particular defect then can be determined by solving Eq. (6). The computation can be simplified further by breaking down the matrix $\Delta$ into symmetry-adapted components. The results are particularly simple for the Ramanactive (gerade) modes. First, since the Raman tensor is effectively symmetric for off-resonance scattering, the $T_{1 g}$ spectrum is absent; and second, since there are no cross terms between gerade modes, the $A_{1 g}, E_{g}$, and $T_{2 g}$ spectra can be treated independently.

If $\Delta=0$, the Stokes components of the Raman spectra provide at $0^{\circ} \mathrm{K}$ a direct map of the $A_{1 g}, E_{g}$, and $T_{2 g}$ spectral functions defined by Eq. (18) when $r=s=1, r=s=2$, and $r=s=4$, respectively. If $\Delta \neq 0$, one can still treat the spectral components independently and vary the symmetry-adapted elements of $\Delta$ to "tune" theory to experiment (as was done by $\overline{\mathrm{HP}}(\mathrm{W})$.

\section{RESULTS}

We have calculated all possible spectral weight functions $A_{r s}(\omega)$ for all of the NaCl-structure alkali halides. This involved calculating all the symmetry-adapted combinations of eigenvectors (SACEV) defined by Eqs. (7)-(17) using a sample of 64000 wave vectors in the first Brillouin zone. The function $A_{r s} \Delta \omega[\mathrm{Eq}$. (18)] was computed by summing the individual contributions for all modes with frequencies between $\omega$ and $\omega+\Delta \omega$ and tabulating the results for 1000 equally spaced bins. For each crystal, octahedral complexes with either positive or negative ions at the center were both considered.

Sample results are shown in Figs. 1-4, which show predicted defect-activated $E_{g}$ spectra for $\mathrm{KCl}$, $\mathrm{KI}, \mathrm{RbCl}$, and $\mathrm{KBr}$, assuming no force-constant changes. In these examples, the defect is assumed to be located at a positive-ion site, thereby enabling direct comparison to be made with the corresponding projected densities of states given by HPW 
which are also shown in the figures.

There is qualitative agreement between the two sets of predictions as regards peak positions and shapes, but detailed agreement is poor. This discrepancy largely reflects the fact that the de formation-dipole models were not adjusted to fit the measured dispersion curves. In spite of this, we believe that over-all agreement is good enough for our SACEV compilations to be useful to re- searchers initiating defect studies and needing firsttrial solutions to use as a basis for subsequent, more refined theoretical studies. Therefore, the tabulated data are available upon request.

\section{ACKNOWLEDGMENTS}

The authors would like to acknowledge extensive discussions and communications with Professor C. W. McCombie and Dr. M. J. L. Sangster.
${ }^{*}$ This work was performed under the auspices of the U.S. Atomic Energy Commission.

$\dagger$ LLL consultant; permanent address: Behlen Laboratory of Physics, University of Nebraska, Lincoln, Neb. 68508.

${ }^{1}$ R. T. Harley, J. B. Page, Jr., and C. T. Walker, Phys. Rev。B $\underline{3}, 1365$ (1971)。

${ }^{2}$ J. R. Hardy, Philos. Mag。 7, 315 (1962).
${ }^{3}$ A. M. Karo and J. R. Hardy, Phys。 Rev. 129, 2024 (1963)。

${ }^{4}$ Generation of an input deck requires the punching of 1000 data cards, the data having been reduced to the optimum bin size consistent with the wave-vector mesh size used in this study。

${ }^{5} \mathrm{C}$. W. McCombie, J. Phys. F $\underline{3}, 351$ (1973). 